\title{
Taxonomy, Polymorphism, and History: An Introduction to Population Structure Theory*
}

\author{
Marc Ereshefsky and Mohan Matthen $\$$
}

\begin{abstract}
Homeostatic Property Cluster (HPC) theory suggests that species and other biological taxa consist of organisms that share certain similarities. HPC theory acknowledges the existence of Darwinian variation within biological taxa. The claim is that "homeostatic mechanisms" acting on the members of such taxa nonetheless ensure a significant cluster of similarities. The HPC theorist's focus on individual similarities is inadequate to account for stable polymorphism within taxa, and fails properly to capture their historical nature. A better approach is to treat distributions of traits in species populations as irreducible facts, explained in terms of selection pressures, genealogy, and other evolutionary factors. We call this view Population Structure Theory (PST). PST accommodates the view, implicit in biological systematics, that species are identified by reference to particular historical populations.
\end{abstract}

1. Introduction. The traditional philosophical approach to natural kinds assumes that they are based on similarity: things belong to a kind if they share certain properties; the kind is defined to capture these similarities. If species and other biological taxa are natural kinds, then the traditional account of kinds needs to be adjusted to accommodate the existence of variation within biological taxa. Some have suggested that the required adjustment is found in the notion of a "homeostatic property cluster" (HPC). Proponents of this view, Richard Boyd (1991, 1999a, 1999b) in particular, hold that while there is no set of properties that all members of a species must share, there is a set of properties that tend to be coin-

*Received April 2004; revised May 2004.

$\dagger$ To contact the authors, please write to: Marc Ereshefsky Department of Philosophy, University of Calgary, Calgary AB T2N 1N4, Canada; e-mail ereshefs@ucalgary.ca. Mohan Matthen, Department of Philosophy, University of British Columbia, Vancouver BC V6T 1Z1, Canada; e-mail: mohan.matthen@ubc.ca.

$\$$ Authors are listed alphabetically. Both authors are pleased to acknowledge (separate) financial support of the Social Sciences and Humanities Research Council of Canada.

Philosophy of Science, 72 (January 2005) pp. 1-21. 0031-8248/2005/7201-0001 $\$ 10.00$

Copyright 2005 by the Philosophy of Science Association. All rights reserved. 
stantiated among the members of a species. These properties, Boyd suggests, are maintained by "homeostatic mechanisms."

We argue that HPC theory is an unsuccessful account of variation within biological taxa. The biological phenomenon of polymorphism poses problems for any similarity-based view, and HPC theory is at heart a similarity-based approach to classification. What is needed, we suggest, is to move away from the focus on the properties individuals share and to take greater notice of populations and other more inclusive entities. These entities are causal actors in the evolutionary process, and they are so in virtue of their phenotype distributions and their population structures. We call this alternative view 'Population Structure Theory' (PST).

In promoting PST, we do not deny the existence of similarities and the homeostatic mechanisms that account for them. But this should not lead one to think that PST is merely a supplement to Boyd's HPC theory. At the level of what is explained, PST shifts attention away from similarities among individual members of a species and focuses on the distribution of traits throughout a species or a population. Sometimes the distribution of traits will be uniform, at other times not: this is beside the point. At the level of explanation, PST notes that homeostatic mechanisms are part and parcel of a suite of mechanisms that cause populations to display phenotype distributions. Similarity is, in other words, a special case of a trait distribution. Thus, PST offers a broader, more fundamental perspective on variation within biological taxa than HPC theory.

In the final part of the paper (Sections 7 and 8), we apply the population structure perspective to account for the identity conditions of taxa. With its focus on similarity, HPC theory misconstrues the ontology of biological taxa. According to biological systematics, taxa are first and foremost historical entities. Similarities may serve as evidence for determining which organisms are parts of a particular taxon, but it is the historical structure of taxa that provides their ontological basis and the ultimate grounds for their identity. Some HPC theorists nod in this direction by talking of taxa having "historical essences" (Boyd 1999b; Griffiths 1999). However, the role they assign these essences is limited to explaining the similarities found among the members of a species. This conception of historical essences is, we argue, inadequate for explaining taxonomic diversity and identity. By focusing on the population and interpopulation structures of taxa, PST provides the appropriate grounds for understanding taxonomic variation and identity.

2. Surface Taxonomy versus Theoretical Taxonomy. Let us start with preDarwinian ideas about natural kinds. It has long been noted that an important type of explanation in biology is routed through biological taxa, species in particular. Why does this bird have black feathers? Because 
it is a crow. Here, the species is posited as an explanans. And it is thought that in order for a species to play this role, the members of the species must be uniform. For the above explanation to work, in other words, crows must generally be things with black feathers.

What sort of unity do kinds like crow possess, and how does this unity figure in explanations like the above? To answer these questions, it is necessary to understand first how biological taxa are identified. Initially they might be identified by reference to observable characteristics. For there is a natural and practical tendency to sort organisms into groups based on the characteristics that are present to our senses. As Ernst Mayr $(1982,252)$ and Scott Atran $(1990,56)$ have noted, there are indigenous peoples in remote communities who are able to sort organisms into groups startlingly coincident with those groups recognized by contemporary biologists. This coincidence indicates that in many cases biological taxa can be identified by careful attention to surface characteristics.

However, there is a philosophical tradition, starting with Aristotle, in which merely observable similarities are insufficient for explanation, for in general such similarities are only sufficient to establish "that something is so," not "why it is so." This crow has black feathers because all crows do: this attempt at explanation fails because it does not tell us why crows have black feathers but only that they belong to a class of organisms all of which do. Invoking the class of crows yields a prediction, but not necessarily an explanation.

What more do we need to get an explanation? According to Aristotle, one must first define a taxon by means of characteristics fundamental to it, and then derive the desired explanandum from such a definition. Such a derivation is meant to ensure that the derived characteristics flow from the defining characteristics and are not merely conjoined with them. The characteristics that figure in the definition must, Aristotle insisted, belong to the kind "universally and of itself," the second clause indicating reference to a supposed causal essence of kinds from which other characteristics flow. To explain why a crow has black feathers, one must identify what properties crows have "of themselves" and derive the possession of black feathers from statements that describe this essence. Given the ephemeral status of feather color (cf. Mill, System of Logic XXII, 5), it may well not be possible to construct such an explanation, but this is how similarities are to be explained, if they are explainable.

Following the successes of the Scientific Revolution, Locke, who agreed with the general thrust of the Aristotelian demand for causal explanations, recommended a somewhat different strategy. Locke agreed that the characteristics of biological taxa are divided into fundamental and derived (see Ayers 1981), the latter being explained in terms of the former. Aristotle's tendency, however, was to identify biological functions as fun- 
damental, and the characteristics that teleologically subserve these as derived. Locke, following the spirit of his time, took mechanistic causation, rather than teleological subordination, to be the prime explanatory relation. This train of thought led him, in accord with the atomism of his time, to the idea that the fundamental things, the origins of mechanistic causation, were unobservable. There is, Locke thought, a hidden reality of atoms that mechanistically explains the evident phenomena we see. Consequently, he posited a hidden "real essence" to explain the evident surface reality of biological taxa. Following Locke, there is now a tradition in philosophy that holds that there are "deep" characteristics (as we shall call them, echoing Chomsky's terminology), which biological science invokes to explain surface, or observable, characteristics across a taxon.

Now, as followers of Darwin, we know that a biological taxon comprises organisms which display a certain variety. Aristotle believed that organisms could differ from the species-norm with regard only to $(a)$ "accidental" characteristics like size and hair color, and (b) "deficiencies," which came to be as a result of imperfect ontogenetic processes (Sober 1980). The Darwinian paradigm abolishes this conception of species-essence. The organisms that constitute a species often constitute a spread, or smear, in quality-space, a "topological neighbourhood" as Russell is supposed to have said (quoted by Ian Hacking 1991). Thus one cannot say that this man is brown because he belongs to Homo sapiens; one can, however, say that his coloring is in a certain range (a range different from black bears, for instance) for this reason.

This is where Boyd's HPC theory comes into the picture. It attempts to provide a more accommodating substitute for the Aristotelian or Lockean picture of causal explanation within biological kinds. Boyd acknowledges that members of a biological taxon are only imperfectly similar. And he tries to provide a substitute for Lockean causal essences suitable to this realization. He explains the imperfect similarity among members of a taxon by "homeostatic" mechanisms - mechanisms that induce conformity. Groupings based on what we have called "surface characteristics" are brought together by certain "causal structures" which ensure that these characteristics occur together. These causal structures include gene recombination, gene transcription, similar selection regimes, common developmental constraints, and other such factors (Boyd 1999b, 165). "Species are defined . . . by . . . shared properties and by the mechanisms (including both 'external' mechanisms and genetic transmission) which sustain their homeostasis" (1999a, 81).

3. The Taxonomic Process: An Initial Characterization. Once we absorb the implications of a view like Boyd's (or Aristotle's or Locke's), we see why it is wrong to think that taxonomy can appeal to surface character- 
istics without the assistance of "theory." Consider, for example, two varieties of hemlock, one a short shrub-like tree found in the mountains, the other a tall tree found at lower altitudes. A classification by surface characteristics alone might assign these to different taxa. However, when it becomes apparent that the environment influences the height and direction of growth, and that offshoots of a plant situated in one environment take on the other form in the other environment, it is recognized that they belong to the same species. Height, then, is not an independent classifier, but one that emerges from something deeper in conjunction with environment. Thus, one could say that hemlocks share the following characteristic: shrub-if-in-mountains-and-tall-tree-if-at-low-altitude, and further that this shared characteristic flows from some "homeostatic mechanism." Thus, this contrived characteristic is important to membership in the species while the shrub characteristic taken by itself is not. The causal diagnosis helps us find an underlying similarity to replace the superficial variety.

Generalizing from this example, we might sketch a taxonomic endeavor in three steps. As a preparatory move, we define a "morphospace" for the purposes of defining taxa on a certain domain of organisms. Morphospace is an $n$-dimensional quality-space defined by the observable variables thought to be relevant to defining a given species, and differentiating it from other species in the locale. The variables need to be weighted to make variations commensurable to their presumed significance and to discount interdependencies. The morphological similarity of two organisms is defined as the inverse of the distance between them in morphospace.

In order to identify biological taxa, we undertake the following procedures.

1. Plotting in morphospace. We examine groupings of specimens in morphospace, identifying clusters and clumps. We propose surface taxa to correspond with these clusters.

2. Explaining morphoclusters. Following Boyd, we attempt to identify the "homeostatic" factors that maintain these surface taxa. These "mechanisms" will include environmental pressures, genetic recombination, genetic copying, and other factors.

3. Reconstituting morphoclusters. We factor the "deep" homeostatic factors into morphospace, reformulating and reweighting the dimensions according to the relations revealed in 2 . At this point, we return to 1 , until no further changes are needed.

As an illustration of this procedure, consider work on the fruit fly species Drosophila persimilis and Drosophila pseudoobscura (Ridley 1993, 388). The flies in these species are morphologically almost indistinguishable. 
An initial plotting in morphospace places these organisms in one cluster. As far as "surface taxonomy" is concerned, this would be the end of the matter. However, when one moves to an explanation of why these flies are similar, a causal pattern emerges that belies the initial morphocluster. We discover two spatiotemporally overlapping but disjoint groups of interbreeding organisms where each group consists of males and females that mate with one another. The initial morphocluster divides into distinct gene pools. The morphological similarity found in the initial specimen pool turns out to be maintained by two separate interbreeding structures. As it happens, these separate structures share a common historical origin and are subject to very similar environmental pressures: this is why members of the two species are similar to one another. Nonetheless, the fact that there are separate homeostatic mechanisms in D. persimilis and $D$. pseudoobscura indicates that underneath their phenotypic clothing, they are distinct taxa. At step three, then, the initial morphocluster is reconstituted - the morphological variables are reweighed - in light of information concerning homeostatic structures, here interbreeding. Notice that in this example, our three-step taxonomic procedure goes in a direction opposite to that found in the hemlock: here, a unified morphocluster gets split apart. The reconstitution of morphoclusters in light of explanatory exploration is common practice in biological taxonomy (Mayr 1982, 281). This procedure is consonant with Boyd's view.

The use of observable characteristics in taxonomy does not diminish the role of theory, for as we have seen the choice and weighting of observable dimensions of morphospace is determined, in part, by theory. Consider the fate of those schools of biological taxonomy that insisted on purely observational taxonomic definitions. Pheneticism and pattern cladism were in part motivated by the desire to expel "theory" from the taxonomic endeavour. Pheneticists, for example, argue that biological theory has been wrong in the past, so to protect taxonomy from such errors no theoretical assumptions should be used in constructing classifications (Colless 1967; cf. van Fraassen 1980). This empiricist ambition, however, cannot be achieved (Ereshefsky 2001, 186). Each organism has an exceedingly large number of traits - too many to record for a classification. Pheneticists must choose which traits to use in constructing classifications. This demands a theory about which traits are more important than others in constructing classifications. So much for theory neutrality. Some theory or set of beliefs is required for constructing classification, even when positing an initial morphocluster.

4. Polymorphism. The philosophical tradition that we have just outlined is correct in its insistence on deep explanatory factors. However, as we shall now argue, recent proponents of this tradition, in particular Boyd 
(1999a, 1999b) and Ruth Millikan (1999), misidentify the phenomenon, and hence the target of explanation. They think that natural kinds, including biological taxa, are united by similarity within the species-population, and this is what needs to be explained by homeostatic mechanisms. And it is undeniably true that there is similarity within biological taxa. Nevertheless, it is equally true that many taxa are characterized by stable and persistent differences. The recent proponents of HPC theory concede what we have learned from Darwin, namely, that there is variation among the organisms that comprise a taxon, even with respect to characteristics that might be used to define that taxon. But apparently they think that variety weakens a group's claim to being a biological taxon and must therefore be ameliorated in some way. According to HPC theory, natural kinds must support induction. So proponents of HPC theory believe that the urgent task in the face of Darwinian variation is to vindicate induction in a suitably weakened form.

Consider what Millikan says about biological kinds. A biological kind does not "as Achilles' horse did and 'run off in all directions' but remains relatively stable in its properties, maintaining its integrity as a kind" (1999, 55). "There are good reasons in nature why one member of an historical kind is like another, hence why inductions are successful over the kind." Exceptions to these inductive generalizations are explained by the fact that "[t]he copying processes that generate [organisms] are not perfect, nor are the historical environments that sustain [biological kinds] steady in all relevant respects" (55-56). For the most part, Boyd (1999a, 82) agrees: "It is also true of biological species, as Millikan indicates it is of many other natural kinds, that their members are often pretty uniform, so that studying a relatively small sample from a species gets information about (almost) all of its members. Of course the variability within species makes such inferences less secure than . . . in chemistry, but . . . it is the shared features . . . which are centrally important."

The problem with this conception of the explanatory task is that polymorphism (the division of a species-population into phenotypically diverse but stable groups) is a central feature of all but the most primitive biological taxa. Polymorphism needs to be explained, not just explained away. In Millikan's account of biological taxa, taxonomic variability is treated as an exception to uniformity, caused by transcription error and environmental instability. This account of variability is reminiscent of what Mayr (1976) calls "typological thinking." Our claim is that these persistent variations need explanation in their own right.

Consider sexual dimorphism. Within any mammalian species, there are pronounced differences between males and females of a species. Males have testes, and females have ovaries. Males produce many, small, motile gametes (sperm); whereas females produce relatively few, large, nonmotile 
gametes (eggs). The development of a zygote occurs within the body of the female and not the body of the male. These differences only scratch the surface of physiological differences, and in many cases behavioral differences, between the males and females of a mammalian species.

Another striking example of polymorphism is found in the life cycles of organisms. The lives of organisms consist of various life stages, and that variation causes striking differences among the members of a species at a time. A May beetle, for example, exhibits quite different morphological and behavioral traits when it is a larva, a pupa, and an imago. A complex and confusing example is that of the slime mold (Bonner 1993). A slime mold begins as a group of spatiotemporally disconnected amoebae. After about two days of life and good nourishment, the amoebae come together to form a translucent slug about a millimetre long. The slug migrates to an appropriate location and then forms a vertical stalk. The top cells in the stalk then turn into spores, which become the amoebae for the next generation of slime molds.

Then there is the polymorphism found in social animals. Ant species contain castes. Queens and soldiers are markedly different from workers. Soldiers in some species can be nearly 200 times the size of workers and come equipped with morphologically different head and teeth. Moreover, each caste has a characteristic life cycle and an age-related polymorphism.

Finally, there is polymorphism due to chance. Organisms of the same species can differ from one another in size, coloration, performance, and appearance due to differences in heredity and chance events in meiosis and development. Such variation is, of course, essential to natural selection.

These examples indicate that uniformity among the members of a taxon should not be privileged when one is interested in understanding the nature of taxa. Polymorphisms confound the grouping of organisms in stage 1 (Plotting in Morphospace) of the taxonomic process outlined above. Caterpillars clearly constitute a clump in quality space that is distinct from butterflies. Nevertheless, they do not constitute a separate species.

5. The Mereological Maneuver. One might try to counter polymorphism with the strategy we applied earlier to the hemlock example - a strategy we will call the "mereological maneuver." Consider polymorphisms of life cycle. It might be said that what we need to consider is not an organismat-a-moment but an organism-over-its-entire-life. Thus it might be that a man-at-thirty is larger than a boy-at-thirteen. Though stage 1 of the taxonomic procedure might render teenage boys members of a different morphocluster than adults, this is a mistake that will be corrected once one realizes that each organism goes through an orderly progression of agerelated stages. Once one arrives at stage 3 (Reconstituting Morphoclusters) 
in the taxonomic process, one understands that the important quality is not size but an age-size profile, i.e., a function from age to size. Agedependent size polymorphism disappears when this profile replaces simple size as the relevant morphospace parameter. Thus, the taxonomic procedure yields a change in the properties being considered: at the beginning size-at-a-moment was considered. Later on, it became apparent that sizeage profile was the more appropriate classifier.

Similarly, it might be thought, it is not the differing characteristics of the sexes, or of castes in an insect species that is important but conditional characteristics of the form, if-a-male-chimpanzee-then- $F$ and if-a-femalechimpanzee-then- $G$. . . , or if-a-soldier-ant-at-lifestage-l-then- $H$ and if-aworker-ant-at-lifestage-m-then-J and . . . Thus, Boyd writes that we can "characterize the homeostatic property cluster associated with a biological species as containing lots of conditionally specified dispositional properties for which canonical descriptions might be something like, 'if male and in the first molt, P' or 'if female and in the aquatic stage, Q"' (1999b, 165).

One needs, however, to be careful how one uses such powerful logical tools: they can become a universal solvent that makes all variation disappear and collapses the entire biological domain into a single morphoclump. For one could account for the differences between bees and apes by a function on some suitably selected variables such as genetic constitution and environmental inputs (if $G^{1}$ and $E^{1}$, then antennae, if $G^{2}$ and $E^{2}$, then hair . . .). And one could then regard the vast network of ecological relationships that constitute the biosphere as a single homeostatic property cluster maintaining polymorphism in "Gaia."

HPC theorists might reply that where there is a single causal mechanism that produces a mereological similarity such as similarity-through-age, the mereological maneuver is plausible. Where the same mechanism is not involved in all, they say, it should not be invoked. This disposes of the merging of bees and apes. The problem with this response is that there is not always a single causal mechanism to underwrite polymorphism. If the mereological maneuver relies on the existence of an underlying causal similarity that produces the whole profile, then sexual dimorphism falls by the wayside. In the case of sexual dimorphism within a species, the HPC theorist would need to find an underlying similarity between males and females that expresses itself differently in different circumstances. But there is no such similarity in mammalian species. Sexual dimorphism within a mammalian species is due to males and females having different chromosomes and different developmental processes. There is no theoretically meaningful similarity under which the variation between the males and females of such species can be subsumed. Variation here cannot be swept away by a swift application of the mereological maneuver. Nor, obviously, can sex differences be ascribed to imperfect copying or other 
failures of homeostatic mechanisms: this would be a reversion to problematic Aristotelian ideas.

A proper approach to taxonomy must recognize such differences. Moreover it should explore the relations that produce and maintain differences. So in addition to Boyd's "homeostatic" mechanisms we need to recognize "heterotic" mechanisms that produce variation, and "heterostatic" mechanisms that maintain it.

6. Explanation and Population Structure. A good example of the kind of explanation that is appropriate for understanding polymorphism is found in E. O. Wilson's (1968) treatment of castes in the social insects. Wilson assumes that the fitness of an insect colony is reduced by its inability to respond effectively to certain sorts of contingency. There are several types of such contingency, and a degree of specialization is required effectively to deal with each of them. This explains why castes are required. However, an optimal mix of castes is also required. If the caste best able to respond to one contingency rises above a certain frequency, the frequency of a caste adapted to a different contingency drops, and the colony's vulnerability to the latter rises above a threshold of acceptable risk. The optimal mix of castes is calculated by figuring out how to keep the combined cost of responding to various contingencies at a tolerable level. Consequently, the way that a population divides into types depends on "the intensity of group selection with reference to behavioral differentiation" (Wilson 1975, $150)$.

The explicit aim of Wilson's theory is to explain the distribution of difference. For example: "[t]he more specialized the caste becomes, and in general the more efficient it becomes, the less will be its representation in the colony. This inverse relation between ability and numbers, which is a consequence of selection at the colony level, is the direct opposite of what would be predicted from selection at the level of individual organisms" (Wilson 1968, 57). Uniformity is a special case of distribution of difference. Wilson's theory tries to explain why some colonies have more and some have less variation. And it tries to explain why some castes occur in small numbers and others occur in large numbers relative to a colony's size. He attributes this kind of specialization to group selection: the claim is that different colonies with different phenotypic distributions competed against one another, and that the more optimal ones won out.

Wilson's appeal to group selection is inessential to our stance here. The important point for us is that the population is an essential player in the explanatory strategy: population variation, not just uniformity, is being explained, whatever the mode of explanation. Not all aspects of polymorphic distributions have to be accounted for by group selection. R. A. Fisher's sex-ratio argument shows that there can be individual selection 
pressures that bring about a rough numerical equality of the sexes. This too is an explanation of population variation, though it appeals only to individual (inclusive) fitness. A central concern of Population Structure Theory (PST) is that polymorphic distributions (the frequencies of stable subtypes within a population) stand in need of explanation. According to PST, polymorphism will often be explained by appealing to those mechanisms that cause heterogeneity and heterostasis, not just those that induce homeostasis. Moreover, PST sees population structure as an important aspect of those mechanisms that produce and maintain polymorphism. Frequency-dependent selection, sexual selection, as well as group selection, all depend on relations among members of a population.

How general is PST? HPC theorists may respond that PST has limited application to a few particular cases. We disagree. Consider polymorphism due to lack of homeostasis. There is (perhaps) a stable proportion of lefthanded humans in Homo sapiens, as there is a stable proportion of different sorts of color-"blindness," different hair-colors, and so on. Further, this stable proportion is geographically well distributed: color-blindness, for instance, is not concentrated in some region where color is ecologically unimportant. Supposing that there is no great difference of fitness among these types, it could be argued that heterosis and heterostasis are not required to explain the proportion of these traits in the population. The gene pool happens to contain this proportion, and Hardy-Weinberg recombination maintains it. From the point of view of HPC theory, this is all there is to it: such "chance" polymorphic distributions do not require PST.

This line of argument in effect treats selection as if it were always a homogenizing influence, and treats chance variation as due to an absence of selection. But this is a mistaken view. Consider two points. The first is that there may be population-level pressures of the sort envisaged by Wilson on conformity and difference. Thus, it is certainly possible that there might be competition among groups on grounds of trait frequency distributions: for instance, a group with greater variance with respect to height might, under certain circumstances, do better (and under others, do worse) than a group with less variance in this respect. There may also be pressures to speciate when these trait frequencies get too spread out. If tall humans prefer each other for mating purposes, and similarly short ones, you could end up with mating barriers between the two groupsreproductive isolation. So one cannot rule out the operation of population-level factors that are generally sensitive to both similarity and difference. That a particular population shows a certain amount of variation with respect to a particular trait might be explained by the strength or weakness of this sort of factor. Moreover, the degree of variation might itself be influenced by selection. Perhaps variation with respect to eye- 
color makes no difference to fitness whereas variation as to number of eyes does. If this is so, one would expect more variation with respect to the former than the latter. Thus, the distribution of eye-color is explained by selection: it is not simply a failure of homeostasis.

The second point is that regardless of the presence of such populationlevel causes, it is a mistake to think that selection is always homeostatic. True, selection often produces a normal distribution (i.e., one that is symmetrically distributed about a single peak). For example, it may be that the faster an organism is the more fit it is, though there are costs associated with being superfast. In this case, there will be a distribution of traits around a median speed value. However, there are other situations in which traits at the two ends of the spread are favored over those in the middle. This phenomenon is known as disruptive selection (Endler 1986, 17-18). Suppose that very slow but strong individuals, and very fast but fragile organisms both have an advantage over those that are moderately fast and moderately strong. Here, variation will increase; the population will tend to fall into a bimodal distribution. There are plenty of realistic cases of disruptive selection: sexual dimorphism is an obvious one. Here, selection favors two divergent phenotypes at the expense of anything "in the middle" and thus maintains diversity. Surveying such cases, one understands why John Endler says: "Natural selection is a process that affects the frequency distribution of heritable traits of a population" (16). Stronger selection does not invariably result in greater uniformity; selection is not always a homogenizing influence. Selection results both in uniformity and in variety.

HPC theorists could respond by downplaying the role of species. The proper neo-Lockean kinds are to be found underneath and across species, it might be argued. Earlier, we discussed and rejected the mereological maneuver by which conspecific males and females were united under a conditional similarity - we argued that this overlooked the diversity of homeostatic mechanisms maintaining the separate sexes. One response to our argument would be that in biology we should look for kinds at lower levels of biological organization than the species. When it comes to sexual dimorphism, we should focus on the two genders of a species rather than the entire species. The vast majority of contemporary male elk share a number of common properties. So the proper explanatory kind should be male elk, rather than elk, HPC theorists may argue. We may call this the "splitting maneuver."

The splitting maneuver could yield the wrong results in some cases. For (as Denis Walsh pointed out in conversation) it could sometimes occur that males of one species might overall be more similar to males of another closely resembling species than to females of its own species. Moreover, the splitting maneuver is fundamentally at odds with explanatory strat- 
egies used by Darwinian systematics. Male elk have many similarities, for example, they tend to have large fuzzy antlers. What explains that similarity? One cause - the proximal cause - of such antlers is the individual development of each male elk. But a deeper explanation-the distal cause - turns on relationships between male and female elks. Arguably, male antlers are the result of sexual selection. Such selection requires the participation of both male and female elk and the utility that each derives in trying to perpetuate individually held as well as shared genes. Looked at in this way, we see that even if we attempt to locate uniform taxonomic kinds below the population or species level, we still must cite those higher levels and the diversity within them. Polymorphism at the higher level (and the attendant population structure that binds that polymorphism) is essential in explaining lower-level similarities within the genders and other subgroups of a species. We have seen that polymorphism cannot be explained away by the mereological maneuver. We see now that polymorphism itself is a vital ingredient for understanding lower-level similarities.

7. Deep Polymorphism. We have gone to some lengths in describing the pervasiveness of polymorphism. Boyd and Millikan embark on the traditional project of explaining uniformity within a taxon. We have highlighted the need to explain diversity within a species - an important desideratum of systematics neglected by many philosophers. We have also discussed how population structure is an important element of that explanation. We would now like to highlight the existence of what we call 'deep polymorphism'. According to the HPC account, homeostatic mechanisms themselves are similarities that unite a species. But as we shall now see, the homeostatic mechanisms of a particular species can also be polymorphic, and what explains such deep polymorphism is population or species structure.

Consider interbreeding within a species and what Mayr calls its "flipside," reproductive isolating mechanisms. Isolating mechanisms prevent members of different species from producing fertile offspring. Such mechanisms can be genetic, physiological, or behavioral. According to Mayr, "the total [reproductive] isolation between two different species is normally due to a great multitude of different isolating forces" $(1963,106)$. Individual organisms often have multiple isolating mechanisms. Furthermore, the organisms within some species have different suites of isolating mechanisms. For example, one organism of a species has isolating mechanisms I1, I2, and I3; another has isolating mechanisms I2, I3, and I4; while a third member has mechanisms I3, I4, and I5. The first and the second organisms can successfully interbreed; the second and third or- 
ganisms can successfully interbreed; but the first and third organisms cannot.

Let us focus on the second type of deep polymorphism, where organisms within a species have different suites of isolating mechanisms. An interesting example is that of ring species (Mayr 1963, 512 ff.). Such species consist of a series of populations geographically spread out over a connected region: over a certain parallel around the globe, or around a continent. Organisms in geographically neighboring populations can interbreed and produce fertile offspring, but organisms from more distant populations cannot successfully produce offspring. According to interbreeding approaches to species, for example Mayr's Biological Species Concept, the populations of a ring species form a single species because they are connected either directly or indirectly by interbreeding. The populations of a ring species form a single gene pool, even though their organisms have different heterostatic and homeostatic mechanisms. In other words, within the big gene-pool there is a complex set of overlapping mechanisms promoting evolution. This inclusive population can therefore be regarded as polymorphic at the deep level as well as the shallow.

Ring species provide an example of synchronic variation among the homeostatic mechanisms of species. There are also examples of diachronic variation among the homeostatic mechanisms of a species. As Splitter (1988) observes, the isolating mechanisms of a species can vary over time. The isolating mechanisms of a species can evolve so that different isolating mechanisms are employed in different generations of a species $(\mathrm{Wu}$ and Ting 2004). If a species forms a single lineage and does not branch, the evolution of that species' isolating mechanisms is open ended. So the best way to describe a species' cluster of homeostatic mechanisms is by using an open-ended disjunction.

In light of such deep polymorphism, one might ask what binds various homeostatic mechanisms together such that they are mechanisms of one species. The answer will not merely cite population structure but some more inclusive structure. In the case of ring species "genetic exchangeability" may be at play (Templeton 1989). Neighboring populations in a ring share genetic material through interbreeding. That genetic material will constrain the evolution of a population's genotypes and isolating mechanisms. As Mayr (1970, 168-169) has argued, new genes and traits in a population must "co-adapt" with the genetic material found in neighboring populations. Each population thus places a constraint on its neighbor's evolution. Each population in a ring species is neighbored by two populations, so the constraint on evolution is from multiple populations. Furthermore, the constraints upon one population by its neighbor are affected by the constraints caused by a neighbor's neighbor, and so on across the links in the ring. Without going any further, we see that to 
understand why the organisms of a ring species have varying isolating mechanisms we turn to the interpopulation structure of a species.

Stepping back, then, we see that polymorphism not only occurs at the level of surface traits of a species but also at the level of underlying mechanisms. Not only is polymorphism at the level of surface traits underappreciated; so is polymorphism at a deeper level. In the examples cited, a proper explanation of deep polymorphism cites interpopulational or conspecific structures. Thus the target of explanation, whether it be surface polymorphism or deep polymorphism, compels us to turn to the population or more inclusive structures of species.

8. Are Historical Essences Historical Enough? The pervasiveness of polymorphism in biological taxa raises a further question. The members of a species vary in their surface traits, and the members of a species are affected by different homeostatic, heterotic, and heterostatic causal mechanisms. In light of such polymorphism, one might wonder why organisms with diverse traits, exposed to a diverse collection of such mechanisms, count as members of a single species. The question here is one of identity conditions of taxa. Most taxonomists appeal to history and genealogy at this point. Quite generally, the thought is that the organisms of a species are genealogically connected and form a distinct lineage on the tree of life. A species' homeostatic, heterotic, and heterostatic mechanisms are mechanisms of one species because they affect organisms that form a unique and coherent lineage.

Supporters of the HPC theory agree that species and other biological taxa are historical entities. Boyd writes that, "It is an important fact that the stability of the property cluster associated with a particular species . . . is entirely a product of historical relations between populations together with external historical contingencies. In this sense species are essentially historical entities" (1999a, 82; see also 1999b, 166). Griffiths (1999, 219 ff.) in his application of HPC theory to biological taxa avers that species are not only genealogical lineages but also kinds with "historical essences." Despite these claims, there is, as we will now argue, a tension in the application of the HPC theory to historical kinds.

Boyd (1999a, 1999b) and supporters of the HPC approach to biological taxa argue that their approach is historical. They describe HPC theory as a departure from traditional accounts of natural kinds that highlight essences found in all and only the members of a kind. On the HPC account, members of a kind need not share a timeless essence. There is no trait or property that all and only the members of a kind must have. Instead, the members of a kind must be sufficiently similar such that successful inductions can be performed. Following Griffiths' $(1999,217)$ description, the similarities among the members of a HPC kind must 
provide a basis for better than chance predictions. This requirement on similarity is a far cry from that required by traditional essentialism. No longer must the members of a kind share a property (or set of properties) that is necessary and sufficient for membership in a kind. A cluster of similarities will do.

Another significant difference between traditional essentialism and the HPC approach is that the mechanisms that cause the homeostatic clustering of similarities need not be intrinsic features of a kind's members. According to traditional essentialism, water's molecular structure or a tiger's DNA are putative essences of their respective kinds: they are intrinsic properties that cause the other properties associated with the members of their kinds. The HPC view allows that kinds may have intrinsic homeostatic mechanisms, but it also allows that kinds may have relational homeostatic mechanisms. The latter mechanisms are particularly apt for species where reproduction is responsible for the continued existence of a species. According to Boyd and other HPC supporters, species have "historical essences" because their organisms have relational homeostatic mechanisms. (We have argued that such relational mechanisms often appeal to population-level factors such as interbreeding.)

This is a significant move in the recent debate over the ontological status of species. Philosophers have tended to treat species as natural kinds where the members of species share a common intrinsic essence. This view goes back to Aristotle and Locke, and is found in the works of Kripke and Putnam. In the 1970s, Ghiselin (1974) and Hull (1978) argued that from a Darwinian perspective biological species are not natural kinds but individuals. Individuals, according to Ghiselin and Hull, are spatiotemporally continuous entities. The organisms of a species form a distinct spatiotemporal lineage whose members are appropriately causally connected. Moreover, the organisms of a species need not share any intrinsic essence. The ability to evolve, both genetically and phenotypically, is a crucial feature of species.

Boyd, followed by a number of authors (for example, Griffiths (1999), Wilson (1999b)), sees the HPC approach as returning species to their rightful ontological place as natural kinds. They agree with Ghiselin and Hull that the organisms of a species need not share an intrinsic property found in all and only the members of a species. They agree that those mechanisms that bind members of a species into a particular species need not be intrinsic; such mechanisms can be relational. What Boyd and HPC supporters bring to the table is that since a species consists of members that share a cluster of homeostatic similarities, species are natural kinds. So supporters of the HPC approach believe that the insights of Darwinism can be accommodated by the HPC view. (Matthen 1998, 119-121, agrees that biological taxa should be treated as kinds, but suggests this can be 
achieved less intrusively, simply by defining them by reference to the historical individuals Ghiselin and Hull have in mind.)

Is the HPC approach sufficiently Darwinian? Boyd clearly intends HPC theory to be true to established scientific practice. Whether a group of entities forms a HPC kind is informed by the scientific theory ranging over the entities in question. Further, the nature of the kind, its homeostatic cluster of properties and its homeostatic mechanisms, is determined by that theory. Given this "naturalist" desideratum, the HPC account of biological taxa should be informed by, or at least be consistent with, biological systematics. But that does not seem to be the case: the historical essentialism of HPC theory is not historical enough for contemporary biological systematics.

Consider Boyd's (1999a, 80) example of a species that arose through multiple hybridization events. Boyd characterizes this species as containing "distinct lineages" that nevertheless have "commonalities in evolutionary tendencies" (80). In other words, the suggested hybrid species contains members with certain similarities, yet the structure of the species is one of spatiotemporally disconnected lineages. The resultant species is not a single historical entity, but a collection of separate lineages with separate origins, but which converge to some extent as a consequence of shared pressures of selection. What is telling in this example is that for Boyd similarity is the final arbitrator of species sameness, not historical connectedness. And this is understandable: how else is he to incorporate both the shared pressures of selection, which are homeostatic and convergent in character, and also the divergence due to lineage. Thus, Boyd writes "I do not, for better or worse, hold that HPC kinds are defined by reference to historical relations among members, rather than by reference to their shared properties" (80). For Boyd, similarity can win out over historical connectedness.

As a view that has the aim of ensuring that kinds have sufficient similarity for induction, the preference for similarity-based kinds over lineagebased kinds makes sense. Yet a taxon of this sort with multiple origins and lacking spatiotemporal continuity - a polyphyletic taxon - is a spatiotemporally disconnected collection of lineages. For the vast majority of biological taxonomists, such polyphyletic taxa are artificial: species and higher taxa are first and foremost historical entities. This assumption is based on the idea that populations are units of evolution. A necessary condition for evolution is that information be passed down from one generation to the next. If species evolve, then the generations of a species must be spatiotemporally connected, otherwise information will be lost. Populations in this sense are fundamentally conduits of information in a lineage. Biological taxa must be nonpolyphyletic groups, otherwise they cannot be properly information carrying. 
Kevin de Queiroz (1999) captures this notion of species in his Lineage Concept of Species. "Species are . . . population-level lineages. This definition describes a very general conceptualization of the species category in that it explains the basic nature of species without specifying the causal processes responsible for their existence or the operational criteria used to recognize them in practice" (53). For de Queiroz, species are not merely lineages of organisms but lineages of populations - a view that is clearly in accord with PST. De Queiroz highlights population lineages to capture the common historical structure found in sexual species and asexual species. De Queiroz's concept is also inclusive enough to allow that some species are monophyletic while other species are paraphyletic, where monophyletic taxa contain all and only the descendants of a common ancestor, and paraphyletic taxa contain some but not all the descendants of a common ancestor. According to de Queiroz, his account captures the core idea of species found in such prominent species concepts as the Biological Species Concept, the Evolutionary Species Concept, and various Phylogenetic Species Concepts. For all of these concepts, species are continuous genealogical entities. (De Queiroz's lineages are individuals, and so, consequently, are his species. However, as noted parenthetically above, this does not preclude the introduction of a species-kind corresponding to membership in the lineage.)

Boyd's application of HPC to biological taxa is at odds with evolutionary approaches to taxonomy. Because he allows similarity to be the overriding criterion for the identification of biological kinds, he permits the existence of polyphyletic taxa. Polyphyletic taxa are considered artificial by the two major schools of contemporary biological taxonomy: cladism and evolutionary taxonomy. Here, then, lies a pressing problem for HPC theory. Boyd attempts to provide an approach to biological taxa that allows substantive decisions to be made by contemporary evolutionary theory. In an attempt to achieve that aim he offers an historical account of biological taxa. Yet that account is not historical enough. Boyd is right that such relational mechanisms as interbreeding and frequencydependent selection can sustain similarities among the members of a species. However, from the perspective of biological systematics, the existence of relational mechanisms is not sufficient for accounting for the historical nature of taxa. Biological taxa must also be single historical entities. This requirement on the ontology of taxa is at odds with HPC theory.

The Population Structure Theory (PST) offered in this paper fully adopts the biological assumption that taxa are first and foremost historical entities. In doing so, PST allows free play to explanatory resources excluded by HPC theory. Suppose, for example, we want to know why certain organisms are members of a particular taxon rather than another. The correct answer turns on whether the organisms in question share a 
common and unique genealogy. It is only when one takes genealogy into account that one can trace the transmission of information down a lineage and distinguish those similarities that are the result of common genetic and developmental stores from those that are not. Only by treating phenotypic distributions as the proper explanatory middle ground between genealogy and individual characteristics can one adequately account for polymorphism. Surface similarities and dissimilarities can provide evidence for species membership. But as we saw in Section 3, such evidence can be deceiving. As Mayr frequently observes, morphological similarity, behavioral similarity, even genetic similarity can only serve as evidence for the identity of a species. Population and genealogical structure are the ultimate determinants of species and taxon membership.

A further identity question that HPC theory does not answer is what causes certain homeostatic mechanisms to be mechanisms of a particular species. In Section 7 we noted the existence of deep polymorphism in species: species consist of a variety of homeostatic mechanisms at a time and over time. According to HPC theory, homeostatic mechanisms cause species to be HPC kinds. But a question, not addressed by HPC theory, is what causes a cluster of homeostatic mechanisms to be mechanisms of a particular species? Again, an essential component of that answer will cite the historical structure of a taxon. A taxon's unique genealogy ties the organisms and causal mechanisms together. Two organisms may be exposed to the same sort of selection pressures, but unless they are bound by genealogy they are not acted upon by the same homeostatic mechanisms. With its focus on similarities, HPC theory fails to properly explain taxon identity. We find it quite striking that HPC theory even fails to explain why a cluster of homeostatic mechanisms are mechanisms of a particular taxon.

Griffiths (1999) offers a version of HPC theory that incorporates aspects of phylogenetic systematics. So at first glance it would seem that Griffiths' HPC account may adequately capture the historical nature of taxa. Following Boyd, Griffiths desires an account of taxa that treats them as kinds of similar organisms - taxa should be categories of organisms that allow for successful induction. The explanatory mechanism Griffiths cites for explaining similarities among the members of a taxon is clearly historical. "[P]hylogenetic inertia is what licenses induction and explanation of a wide range of properties - morphological, physiological, and behavioural - using kinds defined by common ancestry" (Griffiths 1999, 220). Phylogeny acts as an inertial force maintaining similarities among the organisms of a taxon until that taxon is exposed to a different selective regime. Thus, Griffiths claims to offer a more robustly historical version of HPC theory.

Still there is the question of whether Griffiths' HPC theory can ade- 
quately capture the historical nature of taxa. Griffiths does not stray from the main target of explanation for HPC theory: similarities among the members of a kind that suffice for prediction and explanation. In his application of HPC theory to biological taxa, no mention is made of the polymorphic nature of taxa. Correspondingly, no mention is made of the heterostatic mechanisms of taxa that maintain such polymorphism. A further concern we have with Griffiths' version of HPC theory is a tension between wanting taxa that are groups of organisms that have projectable similarities and treating taxa as phylogenetic entities. In Griffiths' explication, such similarities are explained by a taxon's phylogeny. However, as we have found in connection with Boyd, similarity and phylogeny can give rise to opposing identity conditions for a taxon. According to a main tenet of HPC theory, if taxa are HPC kinds then they must be groups of organisms with projectable properties. If the cluster of projectable properties is lost, then the HPC kind no longer exists. Yet according to cladists, a taxon can have radically different traits and still be the same taxon, so long as phylogenetic integrity is maintained (Ridley 1989). Griffiths' attempt to incorporate cladism in HPC theory is at odds with cladism itself. If biological taxa are to be properly understood, their historical nature must be put first.

The HPC theory may work well when taxa consist of surface similarities explained by similar causal homeostatic mechanisms. But at best that is only part of the nature of biological taxa. Introduce variety at either the surface level or the level of explanatory mechanisms and HPC theory fails to provide an adequate account of taxa. Fortunately, there is a ready account for the nature of taxa that cites the population and genealogical structures of taxa. Population Structure Theory is more consistent with biological systematics and it provides the proper resources for understanding the nature of taxa. Moreover, it is not hampered by a philosophical mandate that attempts to squeeze biological taxa into a philosophical shoe that does not fit.

\section{REFERENCES}

Atran, Scott (1990), Cognitive Foundations of Natural History: Towards an Anthropology of Science. Cambridge: Cambridge University Press.

Ayers, Michael (1981), "Locke versus Aristotle on Natural Kinds", Journal of Philosophy 78: $247-272$.

Bonner, John (1993), Life Cycles: Reflections of an Evolutionary Biologist. Princeton, NJ: Princeton University Press.

Boyd, Richard (1991), "Realism, Anti-foundationalism, and the Enthusiasm for Natural Kinds", Philosophical Studies 61: 127-148.

(1999a), "Kinds, Complexity and Multiple Realization: Comments on Millikan's 'Historical Kinds and the Special Sciences"', Philosophical Studies 95: 67-98. (1999b), "Homeostasis, Species, and Higher Taxa", in R. Wilson 1999a, 141-186. 
Colless, Donald (1967), "An Examination of Certain Concepts in Phenetic Taxonomy", Systematic Zoology 16: 6-27.

de Queiroz, Kevin (1999), "The General Lineage Concept of Species and the Defining Properties of the Species Category", in R. Wilson 1999a, 49-90.

Endler, John (1986), Natural Selection in the Wild. Princeton, NJ: Princeton University Press.

Ereshefsky, Marc (2001), The Poverty of the Linnaean Hierarchy: A Philosophical Study of Biological Taxonomy. Cambridge: Cambridge University Press.

Ghiselin, Michael (1974), “A Radical Solution to the Species Problem”, Systematic Zoology 23: $536-544$.

Griffiths, Paul (1999), "Squaring the Circle: Natural Kinds with Historical Essences", in R. Wilson 1999a, 209-228.

Hacking, Ian (1991), “A Tradition of Natural Kinds", Philosophical Studies 61: 109-126.

Hull, David (1978), "A Matter of Individuality", Philosophy of Science 45: 335-360.

Matthen, Mohan (1998), "Biological Universals and the Nature of Fear", Journal of Philosophy 95: 105-132.

Mayr, Ernst (1963), Animal Species and Evolution. Cambridge, MA: Harvard University Press.

(1970), Populations, Species, and Evolution. Cambridge, MA: Harvard University Press.

(1976), Evolution and the Diversity of Life. Cambridge, MA: Harvard University Press.

_ (1982), The Growth of Biological Thought. Cambridge, MA: Harvard University Press.

Millikan, Ruth (1999), "Historical Kinds and the 'Special Sciences", Philosophical Studies 95: 45-65.

Ridley, Mark (1989), "The Cladistic Solution to the Species Problem", Biology and Philosophy 4: 1-16. (1993), Evolution. Oxford: Blackwell.

Sober, Elliott (1980), "Evolution, Population Thinking and Essentialism", Philosophy of Science 47: $350-383$.

Splitter, Lawrence (1988), "Species and Identity", Philosophy of Science 55: 323-348.

Templeton, Alan (1989), "The Meaning of Species and Speciation: A Genetic Perspective", in Daniel Otte and John A Endler (eds.), Speciation and Its Consequences. Sunderland, MA: Sinauer, 3-27.

van Fraassen, Bas (1980), The Scientific Image. Oxford: Clarendon Press.

Wilson, Edward O. (1968), "The Ergonomics of Caste in the Social Insects", American Naturalist 102: 41-66.

_ (1975), Sociobiology: The Abridged Version. Cambridge, MA: Harvard University Press.

Wilson, Robert A. (ed.) (1999a), Species: New Interdisciplinary Essays. Cambridge, MA: MIT Press.

(1999b), "Realism, Essence, and Kind: Resuscitating Species Essentialism?", in R. Wilson 1999a, 187-208.

Wu, Chung-I, and Chau-Ti Ting (2004), "Genes and Speciation”, Nature Genetics 5: 114 122. 